\title{
DEEP METALLIC SURFACE DEFECT DETECTION
}

\author{
K. Sunitha \\ Assistant Professor, Department of Computer Science and Engineering, \\ Mahatma Gandhi Institute of Technology, JNTUH University, \\ Hyderabad, Telangana, India \\ S. Manish Reddy \\ B.Tech $8^{\mathrm{TH}}$ Semester, Department of Computer Science and Engineering, \\ Mahatma Gandhi Institute of Technology, JNTUH University, \\ Hyderabad, Telangana, India
}

\begin{abstract}
Imperfection Detection on any metallic surface is a critical and imperative interaction to control the characteristics of any items in the business. By the by, because of limited information scale and class of deformities, existing imperfection datasets are not accessible for the position of the indistinguishable model. There are numerous identification moves that are poor in proficiency and precision. In this paper, we have made our own neural organization model and furthermore stacked the VGG-16 model with the ImageNet loads. This model can manage absconds with various scales. Finally, the wide preliminaries on two datasets show that the proposed strategy is generous and can meet accuracy necessities for metallic surface defect distinguishing proof.
\end{abstract}

Keywords: metallic surface, VGG-16, Neural Networks, defect detection

Cite this Article: K. Sunitha and S. Manish Reddy, Deep Metallic Surface Defect Detection, International Journal of Electrical Engineering and Technology (IJEET), 12(5), 2021, pp. 28-37.

https://iaeme.com/Home/issue/IJEET?Volume $=12 \&$ Issue $=5$

\section{INTRODUCTION}

As of late, the requirement for imperfection location frameworks that work during the creation period of assembling has been expanding. Quality control systems are utilized to distinguish the deformities in items and to decide the areas of these blemished territories. Imperfection discovery is both significant and important in assembling on the grounds that delivering defective items can bring about generous monetary misfortunes. Albeit human vision is as yet the customary strategy utilized for this reason during creation, profound learning procedures are more viable than human identification frameworks. There are a few models accessible to recognize metallic surface mistakes on the planet out. However, they are principally used to 
distinguish the kind of imperfection was it and yet couldn't find the inadequate part and furthermore the dataset was insignificant to the point that the calculations before are prepared on that can't be utilized in reality climate so that there can be an issue.

Detect the defects from the metallic surface and we will try to localize the location of the defects on the metallic surface. Localization means adding of the particular bounding box around the defect areas on the metallic surface. And also we are using around 10 classes of different defect problems on the metallic surfaces and the metallic surface includes steel, iron..etc. As there is minimal data available and we merged different datasets we need to make proper balanced dataset so that there can be no max priority on it. We also perform some basic augmentation techniques to get the better result and our algorithm needs to be exception to produce the better result by small amount of data.

\subsection{Existing System}

There are several models available to detect the metallic surface errourness in the world out. But they are mainly used to detect the what type of defect was it and but not to locate the defect part. And also the dataset was so minimal that the algorithms before are trained on that cannot be used on the real world environment so that there can be issue. They have less accuracy. The previous systems was trained on the small amount of defects.

\section{Disadvantages}

- They may not give the real world results on the small amount of datasets.

- a low and imbalanced amount of data is available.

- The model doesn't locate the exact defect area on the metallic surface, they just classify the defect on the surface of the metal

\subsection{Proposed System}

The proposed model focuses on increasing the datasets by combining the different datasets together. And maintaining the stability among the classes. And we also perform the special augmentation techniques in order to enhance more the dataset in some of the classes to maintain the stability, for augmentation we use a techniques of flipping, rotating. etc.

We are using a Neural Network model so that it can not only classify the defect but also to locate the exact coordinate and draw a square box around the defect area so as to locate the erroneous. It can help the industries to automate the task to an system it can classify as well as they can find location and help to remove the defect by chopping or other techniques.

\section{Advantages}

- Able to produce the best result on the limited amount of dataset.

- Helps in locating the exact coordinates of the affected area.

- performing the augmentation techniques and increasing the dataset. 


\section{SYSTEM ARCHITECTURE}

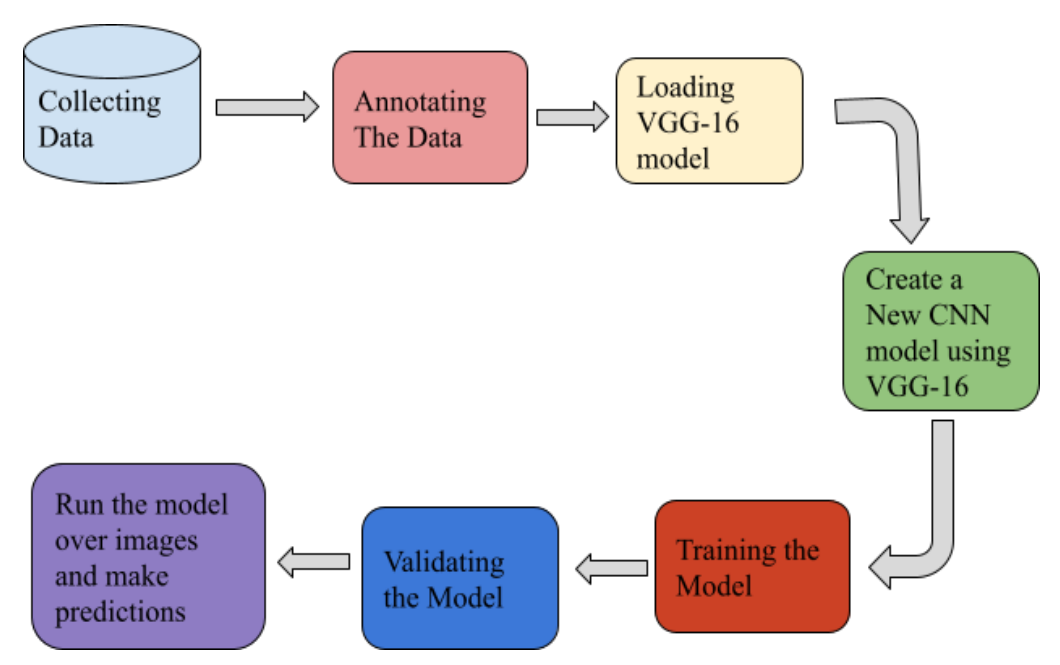

Figure 1 System Architecture

The above figure: 1 system design portrays the means in developing a model for which it can distinguish the imperfections on the metallic surface and furthermore discover which kind of blunder class the deformity has a place with. In the information comment stage, we will attempt to comment on the pictures with the directions for which the deformities or on the pictures. And further more at the substance of the exchange learning stage, we will attempt to stack the exception model so we can utilize their loads on them with the goal that we can great outcomes by utilizing an insignificant measure of information. At that point, we apply MaskRCNN to get the limitation result on the pictures.

What's more, we likewise train on the pictures utilizing the R-CNN model, and from that point onward, we approve the outcome on every single age. Later the model will be saved with the goal that it tends to be utilized for future purposes.

\section{DEEP LEARNING TECHNIQUES}

\subsection{VGG-16}

VGG is an abbreviated structure for the Visual Geometric Group from Oxford University and VGG-16 is a relationship with 16 layers proposed by the Visual Geometric Group. These 16 layers contain as far as possible and there are different layers besides like the Max pool layer yet those don't contain any serviceable cutoff points. This plan was the principle sprinter up of the Visual Recognition Challenge of 2014 for example ILSVRC-2014 and was made by Simonyan and Zisserman.

Underneath shown figure 3.2 is the general organization arrangement of various models made by VGG that utilizes a similar guideline however just shifts top to bottom. we can consider that to be the model moves from more straightforward to complex the profundity of the organization is getting expanded. This is the most ideal approach to take care of any issue, which intends to say, take care of the issue utilizing a more straightforward model and afterward step by step enhance it by making it complex.

\section{Architecture of VGG-16}

In figure :2 over, every one of the blue square shapes address the convolution layers alongside the non-straight actuation work which is a redressed direct unit (or ReLU). As can be seen from 


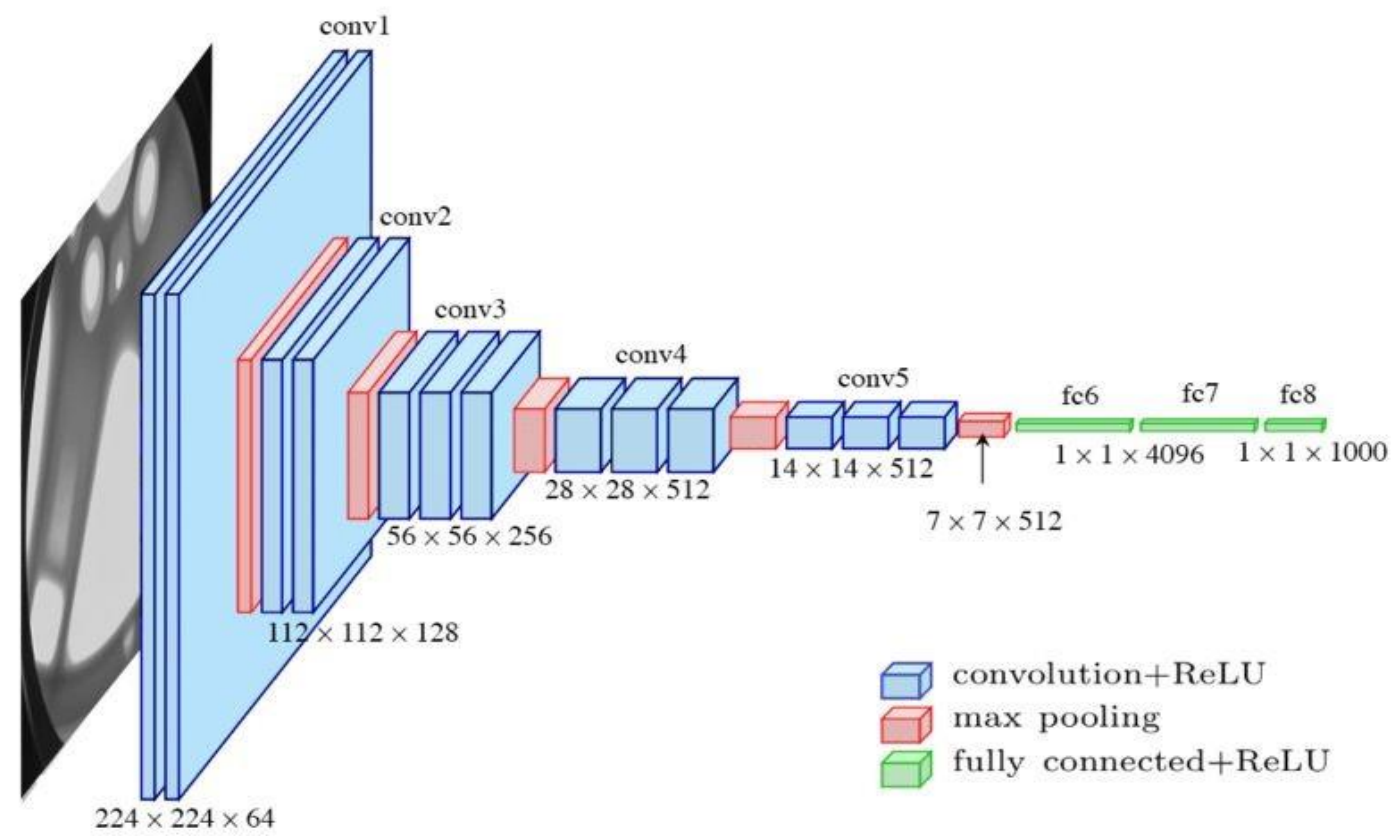

Figure 2 Architecture of VGG-16

figure: 2 that there are 13 blue and 5 red square shapes i.e there are 13 convolution layers and 5 max-pooling layers. Alongside these, there are 3 green square shapes addressing 3 completely associated layers. Along these lines, the complete number of layers having tunable boundaries is 16 of which 13 is for convolution layers and 3 for completely associated layers, consequently the name is given as VGG-16. At the yield, we have a softmax layer having 1000 yields for every picture classification in the imageNet dataset.

The flattened architecture of VGG-16 is as shown below:

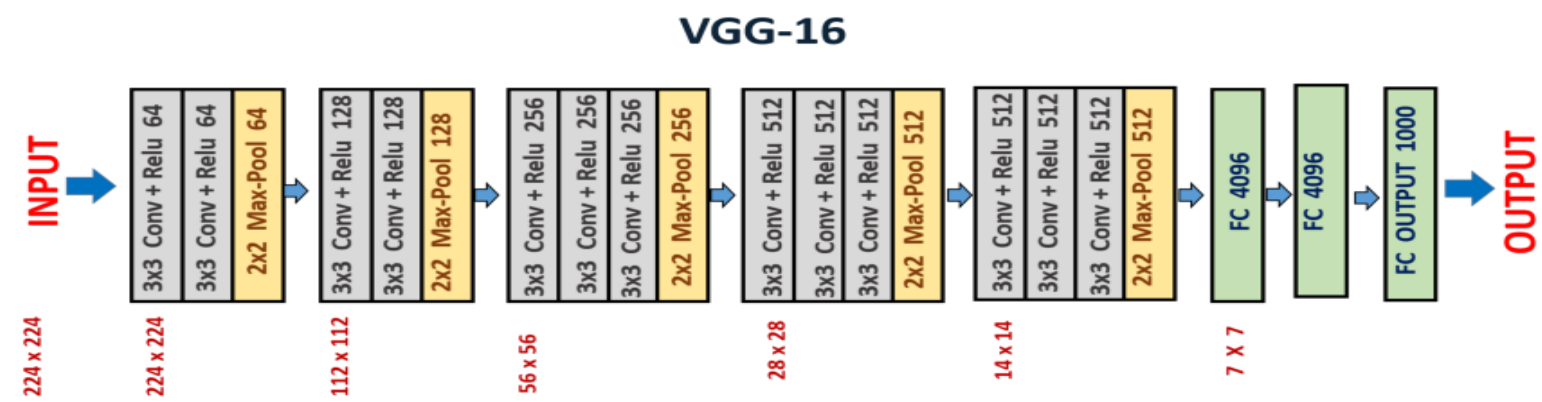

Figure 3 Flattened architecture of VGG-16

In the above figure 3 , the design portrayed is basic. It has 2 touching squares of 2 convolution layers followed by a maximum pooling, at that point it has 3 adjoining squares of 3 convolution layers followed by max-pooling, and finally, we have 3 thick layers. The last 3 convolution layers have various profundities in various structures.

\section{MODULE DESCRIPTION}

\subsection{Dataset}

The GC10-DET dataset is available on the github. GC10-DET is the surface defect dataset collected in a real industry. It contains ten types of surface defects, i.e., punching $(\mathrm{Pu})$, weld line (Wl), crescent gap (Cg), water spot (Ws), oil spot (Os), silk spot (Ss), inclusion (In), rolled 
pit (Rp), crease (Cr), waist folding (Wf). The collected defects are on the surface of the steel sheet. The dataset includes 3570 gray-scale images.

Punching: In the creation line of the strip, the steel strip should be punched by the item determinations; mechanical disappointment may prompt undesirable punching, bringing about punching absconds.

Welding line: At the point when the strip is transformed, it is important to weld the two loops of the strip, and the weld line is created. Stringently talking, this isn't a deformity, however it should be naturally distinguished and followed to be dodged in resulting cuts.

Crescent gap: In the creation of steel strips, cutting here and there brings about abandons, actually like a large portion of a circle.

Water spot: A water spot is delivered by drying underway. Under various items and cycles, the necessities for this imperfection are extraordinary. Notwithstanding, in light of the fact that the water spots are by and large with low difference, and are like different imperfections, for example, oil spots, they are generally recognized unintentionally.

Oil spot: An oil spot is normally brought about by the defilement of mechanical oil, which will influence the presence of the item.

Silk spot: A nearby or consistent wave-like plaque on a strip surface may show up on the upper and lower surfaces, and the thickness is lopsided in the entire strip length bearing. By and large, the principle reason lies in the lopsided temperature of the roller and the lopsided pressing factor.

Inclusion: Incorporation is a normal imperfection of metal surface deformities, ordinarily showing little spots, fish scale shape, strip shape, block sporadic dispersion in the segment of the upper and lower surface (worldwide or nearby), and is regularly joined by unpleasant scarred surfaces. A few incorporations are free and simple to tumble off and some are squeezed into the plate.

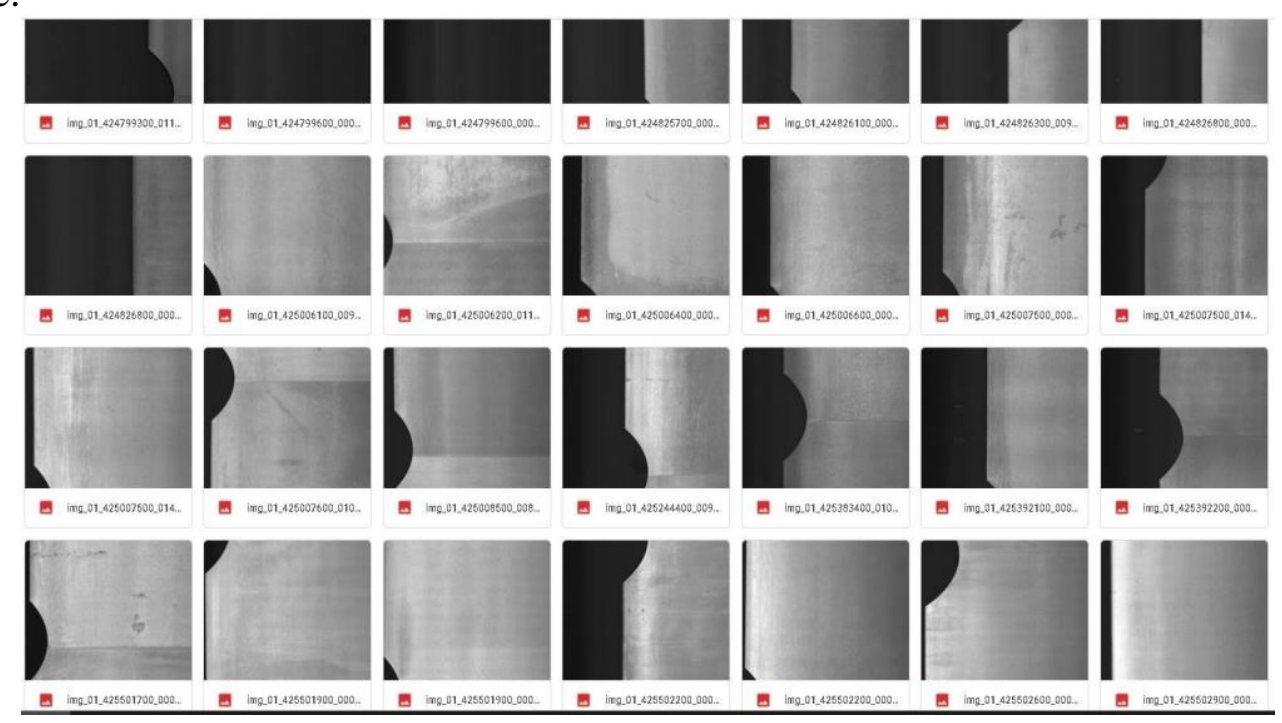

Figure 4 Sample Dataset

Rolled pit: Moved pits are occasional lumps or pits on the outside of a steel plate that is punctate, flaky, or strip-like. They are circulated all through the strip length or segment, chiefly brought about by work roll or strain move harm.

Crease: A wrinkle is a vertical cross over crease, with standard or sporadic dividing across the strip, or at the edge of the strip. The fundamental explanation is the nearby yield alongside the moving heading of the strip in the uncoiling interaction. 
Waist folding: There is clear overlap in the imperfection parts, somewhat more well known, similar to wrinkles, demonstrating that the nearby distortion of the deformity is excessively huge. The explanation is because of low-carbon.

The above figure 4 shows some of the demo images of some classes. These are a kind of $\mathrm{x}$ ray images so that we can even find some air cracks in them.

\subsection{Dataset Preprocessing}

Before starting the training of the model, we need to make sure that the data is preprocessed correctly so that the training of the model can be done without any interruptions.

Here we need to find out the exact dimensions of the bounding boxes from the annotations and the images of the dataset so that our model can know what to identify from the image and what to neglect.

\section{Data Annotation}

Annotating data means combining the data images with the external data like Bounding box coordinates and also the other details to the particular image so that we can train and use them to get the output.

\section{Data Augmentation}

We have performed some data augmentation techniques such as Flip, rotate, brighten by using these techniques. We have increased our data set for the 4 Times. And the coordinates of the bounding boxes are done with the same operations so that it can't have any issue with the coordinates.

\section{MODEL IMPLEMENTATION}

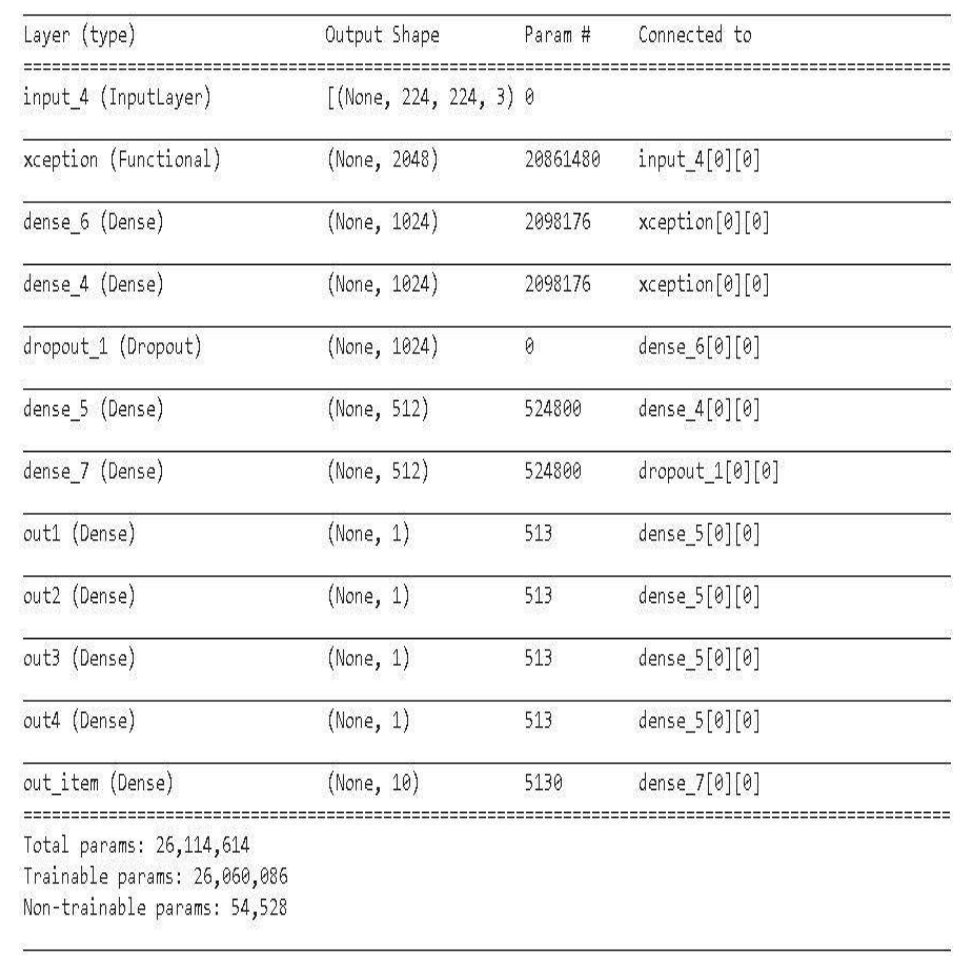

Figure 5 Remodified model

For getting a small amount of data, we have taken a best model and optimized it to our needs and then used it on our training data. Here we have used the VGG-16 model also with 
the model we have added 5 more layers to get the result accordingly to our needs because we have taken the last layer that is with 10 neurons (Dense layer) and before that we have 4 layers each predict the 4 neurons as output i.e 4 coordinate $(\mathrm{x} 1, \mathrm{y} 1) \&(\mathrm{x} 2, \mathrm{y} 2)$, which are the diagonal elements of the bounding box of the coordination. We are not training from the scratch but we are loading the weights of the imageNet and trying to train on those weights, so that we can get better accuracy faster on proper usage of the resources. In this model we can also improve our accuracy using the hyper parameter tuning.

\section{ERROR EVALUATION}

\subsection{Mean Squared Error}

MSE is the normal of the squared blunder that is utilized as the misfortune work for leastsquares relapse. It is the aggregate, in general, the information focuses, of the square of the contrast between the anticipated and genuine objective factors, partitioned by the quantity of information focuses.

$$
\mathrm{MSE}=\frac{1}{n} \sum_{i=1}^{n}\left(Y_{i}-Y_{i}^{\Lambda}\right)^{2}
$$

Equation 1: MSE Error Formula

MSE=Mean Squared Error

$\mathrm{n}=$ number of data points

Yi=Observed values

$\mathrm{Y}^{\wedge} \mathrm{i}=$ Predicted values

\section{TESTING}

In this paper we are training over 1824 images and validated over 456 images and tested on 50 images. we take input size as $(224,224,3)$. Output the 4 coordinates and the classes it belongs to.

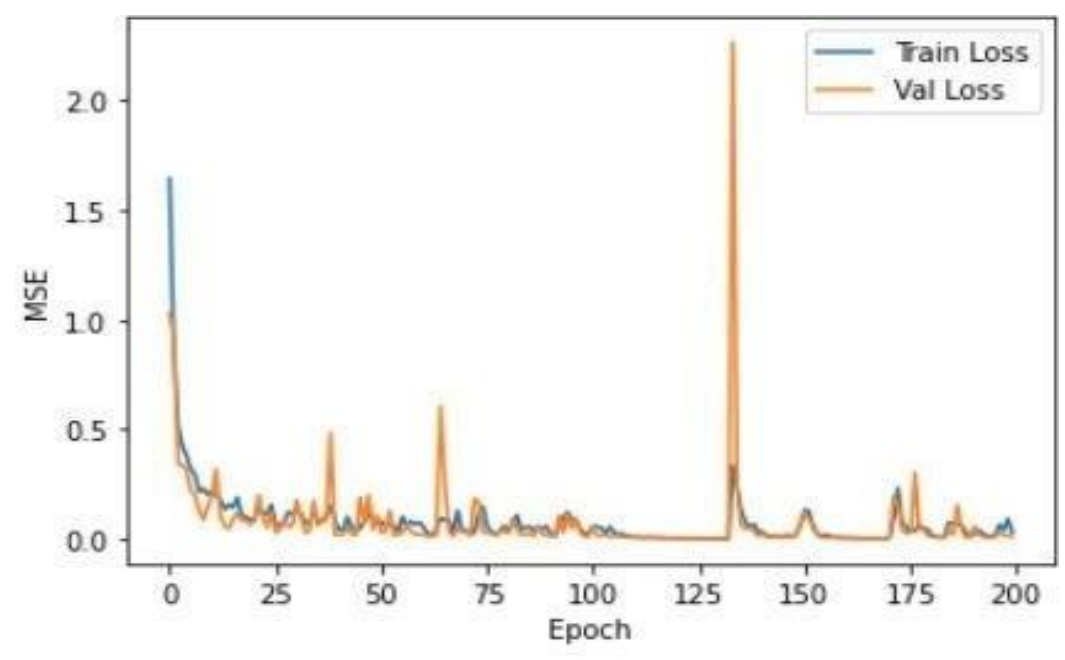

Figure 6 Error data graph over each epoch

In the above graph we are having Mean Squared error(MSE) represented for each epoch for the from 0 to 200.Here we can see the there is a gradual decrease in the Error rate in both training and validation. But we can see there is a huge curve going upwards over the 127 epoch it states that there may be a new classes introduced to the network so getting the new thing as an input it is unable to find the result and the error has increased compared to the previous epochs. But as the training goes on the training the MSE is reduced to 0.02. 


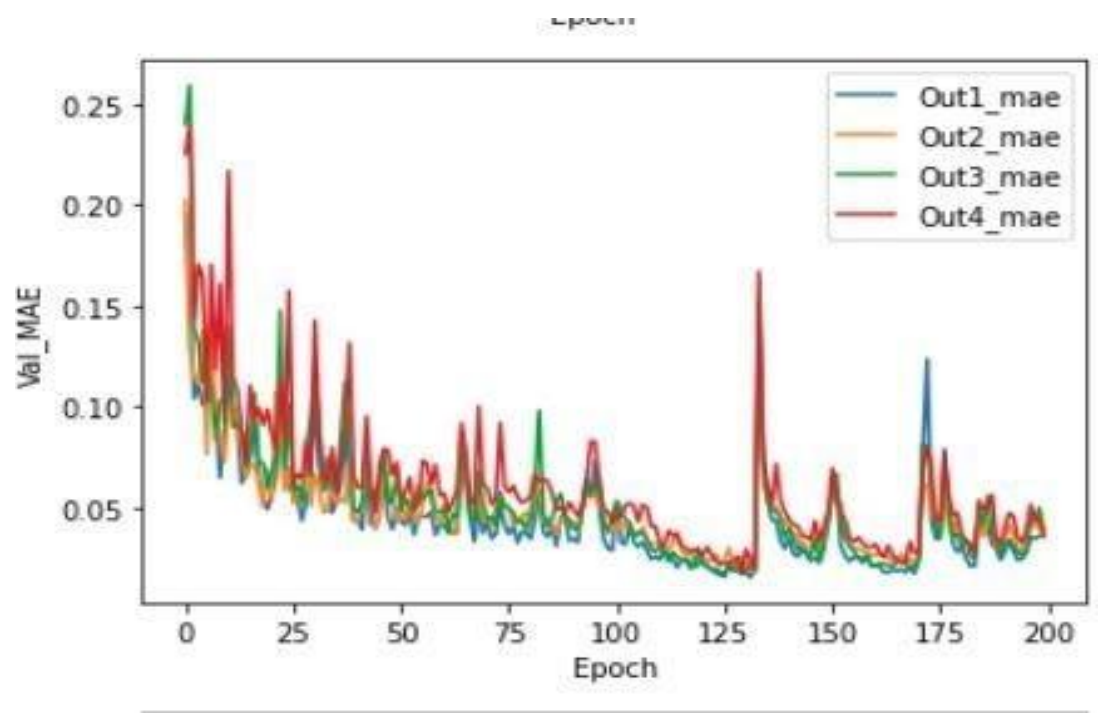

Figure 7 Out1,Out2,Out3,Out4 MSE over each epoch

In the Fig:7 graph we are using a graph to represent the 4 outputs they are Out1,Out2,Out3,Out4 are generating by testing on validation over the each epoch. The Out1,Out2 represent the $(\mathrm{x} 1, \mathrm{y} 1)$ and the Out3,out 4 represent the $(\mathrm{x} 2, \mathrm{y} 2)$, these coordinates represent the two diagonal parts of the box helps to draw them out.

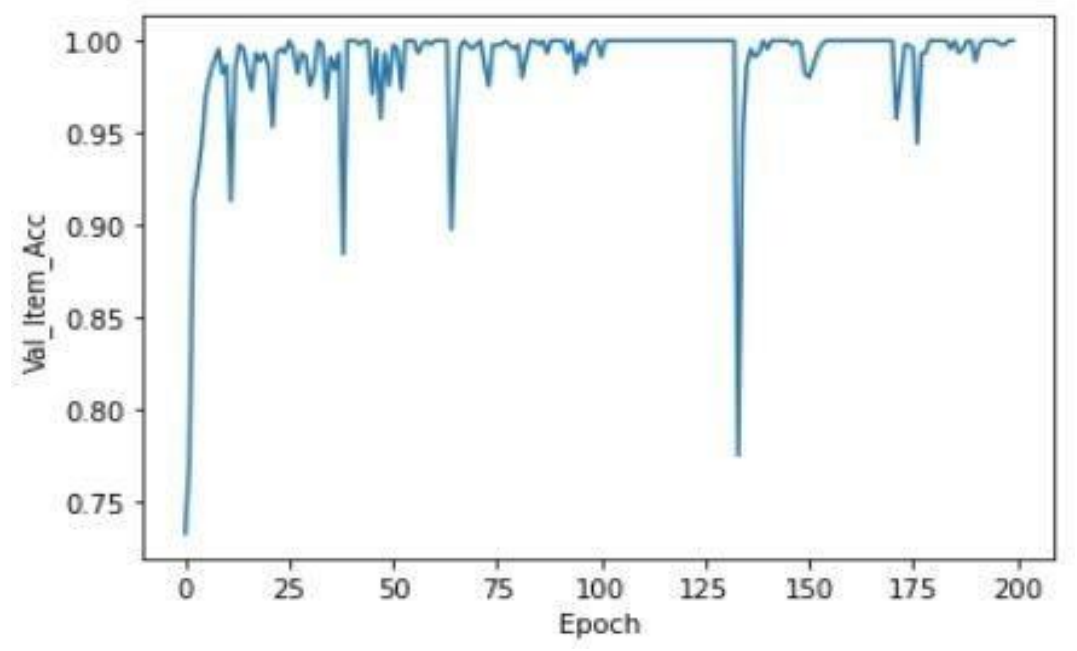

Figure 8 Validation over the each epoch

here we try to train the validation set over the trained data for each epoch and try to represent the accuracy and on Figure 4.3 we can see the accuracy over then are goes on increasing for each epoch and goes upto $97 \%$ accuracy.

\section{RESULTS}

As this paper is about detecting defects on the metallic surface using CNN. We have taken a neural network model loaded with VGG16 and trained over 200 epochs. With the lowest amount of data we have achieved the around $97 \%$ percent of accuracy. The result will give the 4 coordinates and also the type of defect on the metallic surface.

The demo images predicted using the trained model 


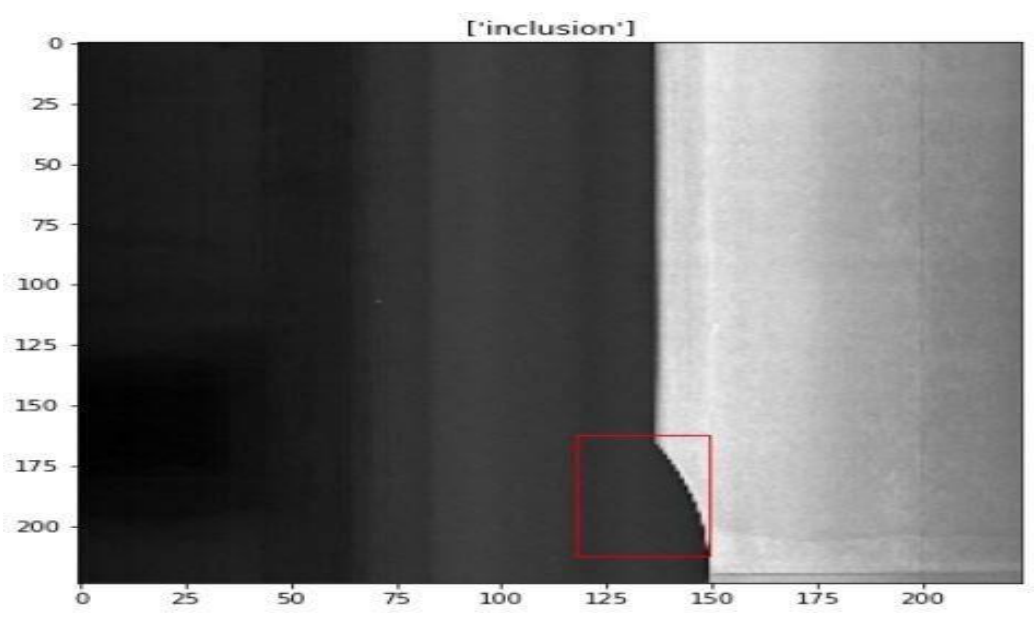

Figure 9(a): Predicted images on the trained model

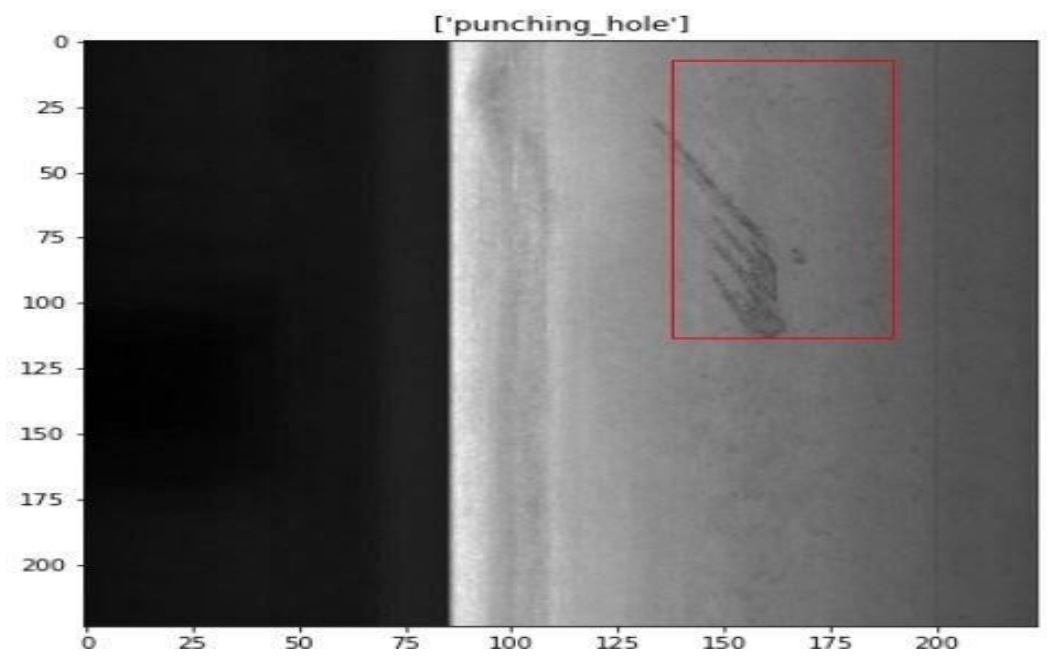

Figure 9(b): Predicted images on the trained model

\section{CONCLUSION}

This paper is about building a deep learning model that helps end to end prediction of the error on the metallic surface. Here we have designed a model in such that it not only classify the class of defect but also helps to localize the exact location of the defect by getting the coordinates as the output. We have also performed some augmentation techniques which help to increase the dataset volume and also helped to train the model even with the noised data. So that it can predict good results even on the heavy noised images.

\section{FUTURE SCOPE}

In future, planning to perform further research and we can perform the improvement in the model and even we can try to build a model so that it not only draw the bounding box but also outline the exact boundary in the error's shape i.e. segmentation, make it usable even in the real world with the normal images and makes the models so flexible that even it can run in the low end systems. 


\section{REFERENCES}

[1] Xian Tao, Dapeng Zhang, Wenzhi Ma, Xilong Liu and De Xu, "Automatic Metallic Surface Defect Detection and Recognition with Convolutional Neural Networks" AIIT,January 2019.

[2] H. Zheng,L.X. Kong,b.S. Nahavandi, "Automatic inspection of metallic surface defects using genetic algorithms" IEEE,September 2017.

[3] Xiaoyang Gai, Peiran Ye, JIngling Wang, "Research on Defect Detection Method for Steel Metal Surface based on Deep Learning",CoRR,April 2020.

[4] Awei Zhou, Han Zheng, Meng Li, Wei Shao, "Defect Inspection Algorithm of Metal Surface Based on Machine Vision", Arxiv,February 2020.

[5] Tiwari Priti Ramesh, Yashoda Bisht; "Detection and classification of metal defects using digital image processing”, IEEE,June 2019.

[6] Liu W., Anguelov D., Erhan D., Szegedy C., Reed S., Fu C.Y., Berg A.C. Ssd, "Single Shot Multibox Detector; Proceedings of the European conference on computer vision", Arxiv, October 2016.

[7] Ren R., Hung T., Tan K.C, “A generic deep-learning-based approach for automated surface inspection”, MDPI, June 2017.

[8] Tastimur C., Yetis H., Karaköse M., Akin E. Rail, "defect detection and classification with real time image processing technique”,Arxiv, October 2016.

[9] Jian C., Gao J., Ao Y., "Automatic surface defect detection for mobile phone screen glass based on machine vision” ,IEEE,March 2017.

[10] Tsanakas J.A., Chrysostomou D., Botsaris P.N., Gasteratos A. "Fault diagnosis of photovoltaic modules through image processing and Canny edge detection on field thermographic measurements",Arxiv,June 2015.

[11] Win M., Bushroa A., Hassan M., Hilman N., Ide-Ektessabi A, "A contrast adjustment thresholding method for surface defect detection based on mesoscopy",AIIT,June 2015. 